\title{
Radio-over-Fiber Transmission Using Vortex Modes
}

Tatarczak, Anna; Lu, Xiaofeng; Rommel, Simon; Rodriguez Páez, Juan Sebastián; Vegas Olmos, Juan José; Tafur Monroy, Idelfonso

Published in:

2015 IEEE International Topical Meeting on Microwave Photonics

Link to article, DOI:

10.1109/MWP.2015.7356677

Publication date:

2015

Document Version

Peer reviewed version

Link back to DTU Orbit

Citation $(A P A)$ :

Tatarczak, A., Lu, X., Rommel, S., Rodriguez Páez, J. S., Vegas Olmos, J. J., \& Tafur Monroy, I. (2015). Radioover-Fiber Transmission Using Vortex Modes. In 2015 IEEE International Topical Meeting on Microwave Photonics IEEE. https://doi.org/10.1109/MWP.2015.7356677

\section{General rights}

Copyright and moral rights for the publications made accessible in the public portal are retained by the authors and/or other copyright owners and it is a condition of accessing publications that users recognise and abide by the legal requirements associated with these rights.

- Users may download and print one copy of any publication from the public portal for the purpose of private study or research.

- You may not further distribute the material or use it for any profit-making activity or commercial gain

- You may freely distribute the URL identifying the publication in the public portal

If you believe that this document breaches copyright please contact us providing details, and we will remove access to the work immediately and investigate your claim 


\title{
Radio-over-Fiber Transmission Using Vortex Modes
}

\author{
A. Tatarczak ${ }^{1}$, X. Lu ${ }^{1}$, S. Rommel ${ }^{1}$, S. Rodriguez ${ }^{1}$, J.J. Vegas Olmos ${ }^{1}$, and I. Tafur Monroy ${ }^{1,2}$ \\ ${ }^{1}$ Department of Photonics Engineering \\ Technical University of Denmark \\ Ørsted Plads, Building 343, Kgs. Lyngby, 2800 \\ Denmark \\ ${ }^{2}$ ITMO University, St Petersburg, Russia
}

\begin{abstract}
This paper demonstrates experimentally the distribution of radio-over-fiber (RoF) signals using orbital angular momentum (OAM) of light over standard OM4 multimode fiber (MMF) at $850 \mathrm{~nm}$ wavelength. Five independent OAM modes are used to convey RoF signals in the microwave regime showing robust performance and therefore opening new prospects for enhancing the capacity of MMF based RoF Links.
\end{abstract}

Keywords- orbital angular momentum; microwave signals; radio-over-fiber; mobile fronthaul

\section{INTRODUCTION}

The development of wireless networks is adding pressure to the transport networks underneath, which had effectively moved from copper based links (see Fig. 1) to fiber based links. Current fiber based distribution systems for wireless, mobile fronthaul, is now experiencing a new phase of regeneration: radio-over-fiber (RoF) systems [1] and free space optics (FSO) [2] have been proposed and successfully demonstrated to provide large bandwidth to antenna sites, smoothly integrating the mobile fronthaul segment with the mobile backhaul segment. The advantages of using RoF techniques to distribute wireless signals are multifold: it provides a scalable technology that allows seamless integration of the optical access network and the transmitting antenna by providing direct optical baseband to optical RF upconversion. The low transmission loss of optical fiber allows for antenna remoting, allowing centralizing wireless carrier generation and signal processing. Photonics is generally of advantage when generating and transmitting spectrally-broad and spectrally-efficient ultra-high capacity data signals. On the other hand, FSO has been proven to be a flexible tool to link short- to mid-range distances [3]. A deficiency in FSO is the under-utilization of the available spectra. Orbital angular momentum (OAM) of light has been proposed to effectively increase the capacity of FSO links by multiplexing several channels using the modes of the light [4], [5]. An alternative usage of OAM modes has been presented for short-range multimode fiber (MMF) links and showed improvement of transmission performance in terms of bit error rate (BER) for higher order OAM modes [6]. The OAM of light is the component of angular momentum of a light beam that is dependent on the field spatial distribution, and not on the polarization. It can be further split into an internal and an external OAM. The internal OAM is an origin-independent angular momentum of a light beam that can be associated with a helical or twisted wavefront. The external OAM is the origin-dependent angular momentum that can be obtained as cross product of the light beam position (center of the beam) and its total linear momentum [7]. By manipulating the OAM modes, multiple light beams can co-exist within the same space without interfering to each other. This effectively increases the capacity of the channel.

Fig. 1 shows a network scenario where FSO with OAM is used to support RoF signal distribution for mobile fronthaul systems. Regular single mode fiber (SMF) connections are used to distribute RoF signals to the cell stations; in case an isolated cell stations needs to be feed remotely through an FSO link, we employ OAM to effectively multiplex Nchannels. At the isolated cell station, each individual OAM mode is recovered and the RoF signal fed to the antenna using multimode fiber (MMF), which is more convenient for low cost installations; MMF permits the use of the $850 \mathrm{~nm}$ communication window, which is extensively use in data center environments and therefore has off-the-shelves components readily available.

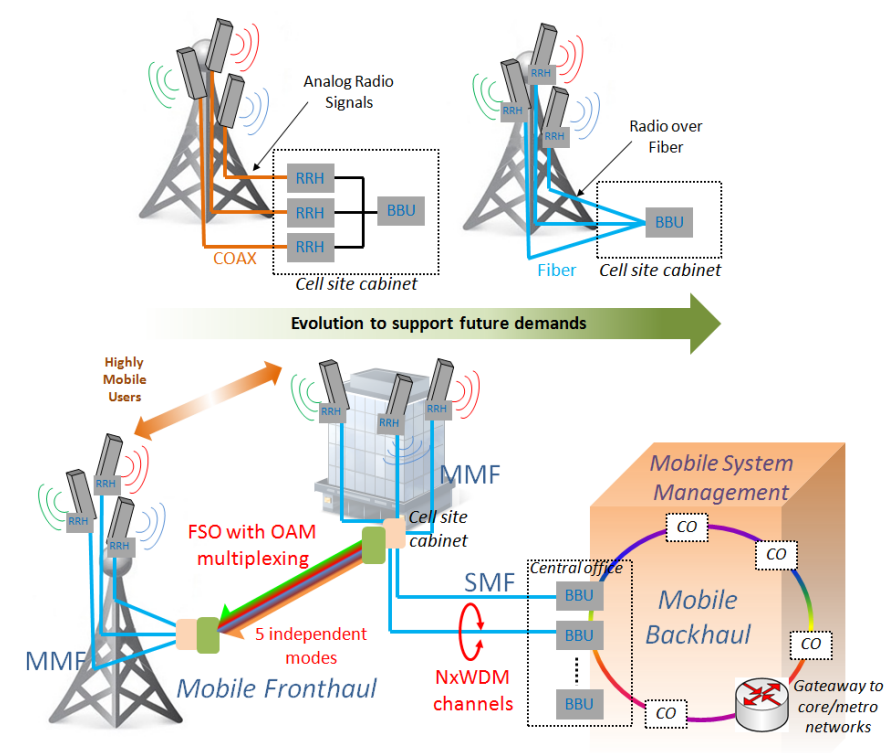

Fig. 1. Evolution from analog radio distribution systems based on copper to radio-over-fiber based signal distribution (upper section). Utilization of orbital angular momentum communications in cell cabinet linkage to overcome physical limitations on the deployment of mobile fronthaul wired infrastructures (lower section). OAM using FSO offers the possibility to 
distribute the channels to cell site cabinets, and later transport the RoF signals to each of the antennas independently using OAM over MMF.

This paper will present experimental results on transmission of radio signal over multimode fiber measured for five OAM modes. The following sections include the experimental setup (Section II) used to demonstrate the proposed technology - a hybrid channel composed of OAM in FSO with fiber distribution, and examination of the main experimental results (Section III) obtained. We will finally discuss the outcome and summarize the results.

\section{EXPERIMENTAL SETUP}

The experimental setup is shown in Fig. 2. A $1.5 \mathrm{Gbps}$ pseudo-random bit sequence (PRBS) is generated by a pulse pattern generator (PPG) and upconverted to a $10 \mathrm{GHz}$ carrier using the external RF mixer of a vector signal generator (VSG). The data sequence length is $2^{15}-1$. The $800 \mathrm{Vpp}$ upconverted signal is used to directly modulate a $850 \mathrm{~nm}$ multimode vertical-cavity surface-emitting laser (VCSEL) biased at $8 \mathrm{~mA}$. The choice of bit-rate and carrier frequency is not based on adhering to any standard but for the purpose of investigating the performance of analog signal transmission over OAM in MMF.



Fig. 2. Experimental setup. PPG: pulse pattern generator, VSG: vector signal generator, VCSEL: vertical-cavity surface-emitting laser, LCOS SLM: Liquid Crystal on Silicon - Spatial Ligt Modulator, PBS: polarization beam splitter, MMF: multimode fiber, PD: photodiode, DSO: digital sampling osciloscope.

The modulated VCSEL beam is launched from the laser's fiber pigtail to free space. In the free space part of the system the multimode beam passes through the polarizer to the spatial light modulator (SLM). The SLM modulates the phase of the incoming beam to helical, creating an orbital angular momentum (OAM) mode. In this work we use SLM to create five consecutive OAM modes: M0-M4, where M0 is a conventional multimode and M1-M4 are OAM modes with orders from 1 to 4 . The optical power level is controlled by the variable attenuator. After a $45 \mathrm{~cm}$ free space path, the beam is coupled into a $1 \mathrm{~m}$ long MMF OM3 patchcord and connected to the multimode fiber spool for transmission performance assessment. The FSO link length is limited by the laboratory space available; fundamentally, the link can be extended, as it has been demonstrated previously [8]. Fig. 3 shows a picture of the free space setup section. We test fiber transmission over $1 \mathrm{~m}$ and $100 \mathrm{~m}$ MMF OM4. The loss in $100 \mathrm{~m}$ spool is 0.37 $\mathrm{dB}$ at $850 \mathrm{~nm}$. The transmitted signal is received with a 850 $\mathrm{nm}$ photodiode with $25 \mathrm{GHz}$ bandwidth, stored at a digital storage oscilloscope (DSO) with $14 \mathrm{GHz}$ bandwidth and downmixed in off-line postprocessing using digital signal processing; no equalization algorithms were employed in this experiment.

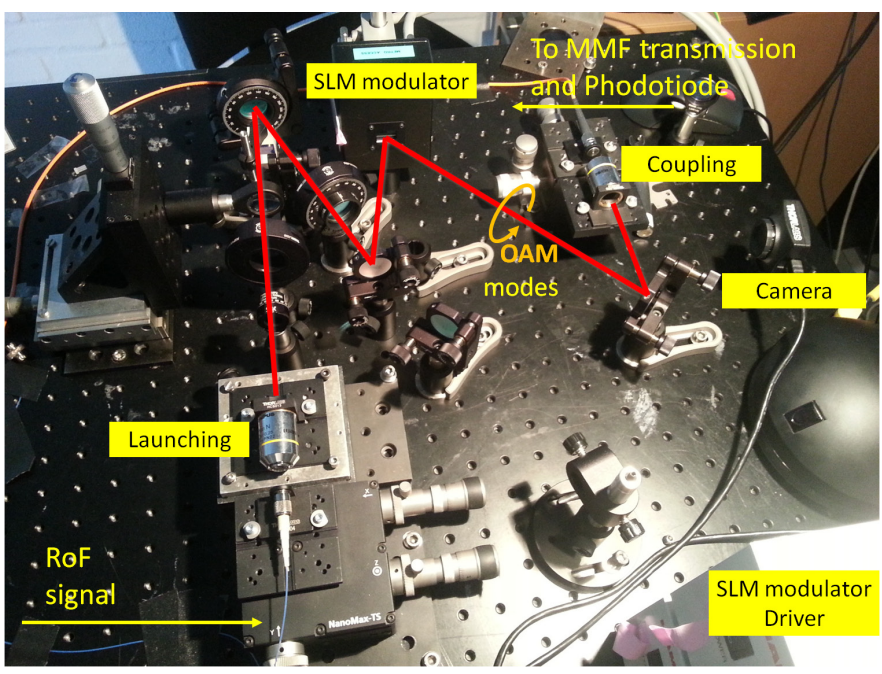

Fig. 3. Free space optics' setup. The wired section of the setup was located in an adjacent optical table. SLM: spatial light modulator.

\section{EXPERIMENTAL RESULTS}

We first assessed the stability of the system when using each of the OAM modes in terms of optical spectra. Fig. 4 shows the optical spectra before and after transmission for each of the five modes with and without RoF modulation. As it can be observed the optical spectra remain stable, as expected, regardless of the OAM used to convey the analog signal and whether the modulation is on or off, as expected.

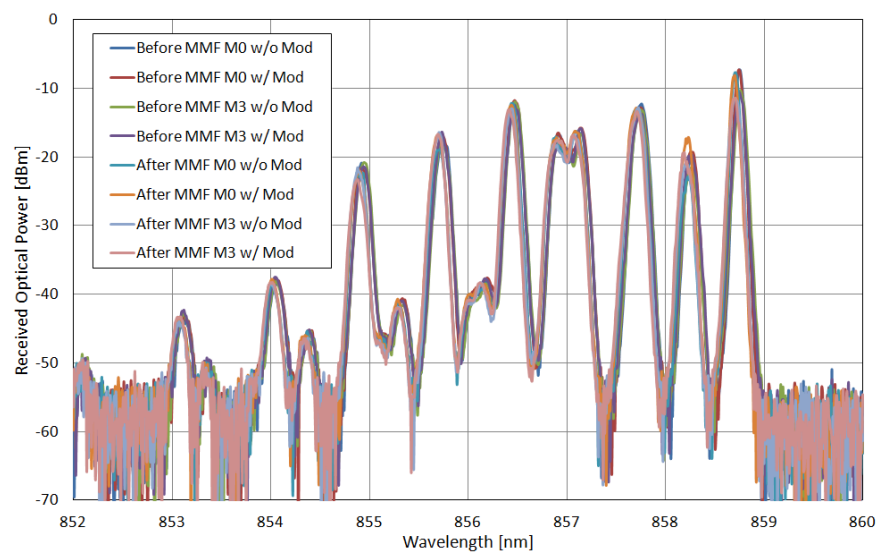

Fig. 4. Optical spectra of each of the modes before and after transmission, and with and withou modulation. As it can be observed, the spectra in all cases remain the same, indicating the stability of the system.

Fig. 5 shows the bit error rate (BER) performance of the system, comprising the measured curves in the back-to-back case and after $100 \mathrm{~m}$ of transmission through OM4 MMF fiber. 


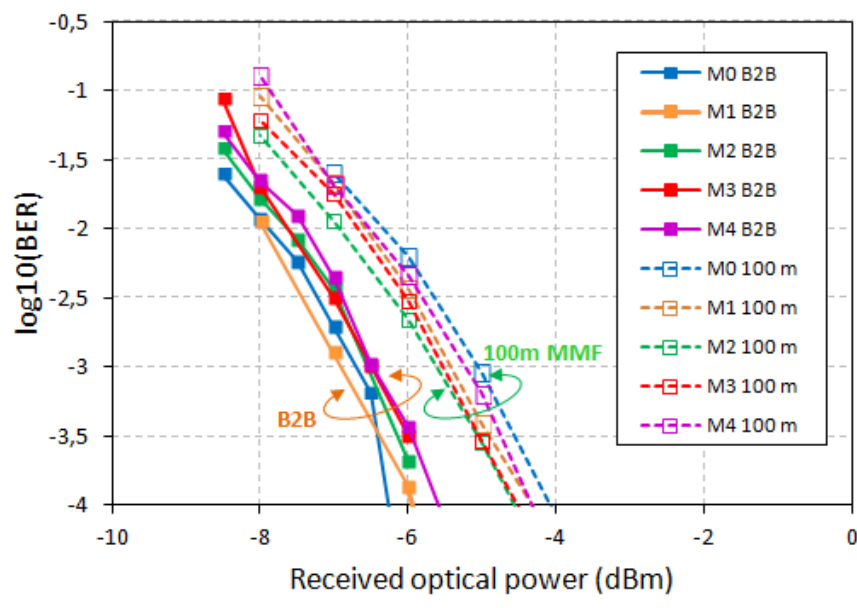

Fig. 5. Measured Bit error rate curves for back-to-back and after $100 \mathrm{~m}$ of OM4 MMF transmission, for each of the five orbital angular momentum modes.

The BER performance for each set of OAM modes in either case remains within the same range, indicating a solid and consistent performance. After transmission, the power penalty at BER of $1 \mathrm{e}-3$ is less than $2 \mathrm{~dB}$ for all cases, indicating again a solid and OAM mode-independent MMF performance of RoF using vortex modes.

\section{CONCLUSIONS}

Transmission of analog signals over conventional MMF fibers with enhanced robustness and enhanced capacity are a new prospect for low cost MMF RoF links. In this work, we demonstrate the solid and stable performace of using up to 5 OAM modes with a carrier signal of $10 \mathrm{GHz}$. Further work includes the study of OAM (de)multiplexing in FSO and the assessment of transmission of more complex modulation formats.

\section{Acknowledgment}

This work was partly supported by the Marie Curie FIWIN5G project, the HOT project of Danish Innovation Fund, by the Government of the Russian Federation (Grant 074-U01) through ITMO Visiting Professorship scheme, and the Villum Fonden SEES project.

\section{References}

[1] K. Kitayama, T. Kuri, J.J. Vegas Olmos, and H. Toda, "Fiber-wireless networks and radio-over-fibre techniques," Conference on Lasers and Electro-optics CLEO, paper CThR4, 2008.

[2] I. I. Kim, E. J. Korevaar, „Availability of free-space optics (FSO) and hybrid FSO/RF systems," Proc. SPIE 4530, Optical Wireless Communications IV, 27 November 2001.

[3] H. A. Willebrand, G. R. Clark, "Free space optics: a viable last-mile alternative," Proc. SPIE 4586, Wireless and Mobile Communications, 11, October 18, 2001.

[4] P. Martelli, A. Gatto, P. Boffi, M. Martinelli, "Free-space optical transmission with orbital angular momentum division multiplexing," Electronics Letters, vol.47, no.17, pp.972,973, August 182011.

[5] Y. Ren, Z. Wang, P. Liao, L. Li, G. Xie, H. Huang, Z. Zhao, Y. Yan, N. Ahmed, M. P. J. Lavery, N. Ashrai, S. Ashrafi, R. D. Linquist, M. Tur, I. B. Djordjevic, A. M. Neifeld, A. E. Willner, "400-Gbit/s free-space optical communications link over 120-meter using multiplexing of 4 collocated orbital-angular-momentum beams," Optical Fiber Communications Conference and Exhibition (OFC), 2015 pp.1,3, 22-26 March 2015.

[6] A. Tatarczak, M. Usuga, and I. Tafur Monroy, "OAM-enhanced transmission for multimode short-range links," SPIE International Society for Optical Engineering, vol. 9390, 2015.

[7] J Wang, J. Y. Yang, I. M. Fazal, N. Ahmed, Y. Yan, H. Huang, Y. Ren, Y. Yue, S. Dolinar, M. Tur, A. E. Willner, "Terabit free-space data transmission employing orbital angular momentum multiplexing," Nature Photonics, 6, 488-496, 2012.

[8] C. Shen, H. Feng, Z. Xu, S. Jin, "High speed free space optical communication system for $1 \mathrm{~km}$ communication," Conference on Lasers and Electro-Optics - Pacific Rim, 2007. 